\title{
Observaciones sobre la Citología del Seno
}

\author{
PRESENTADO EN LA ACADEMIA NACIONAL \\ DE MEDICINA CAPITULO DE BUCARAMANGA \\ EN SESION DEL 18 DE ABRIL DE 1989
}

Dr. Carlos Cortés Caballero*

\section{INTRODUCCION}

Hace algunas semanas tuve la honrosa distinción de participar en unas charlas organizadas por la Sociedad Santandereana de Obstetricia y Ginecología sobre Displasias Mamarias y aunque específicamente no se habló sobre la metodología en el diagnóstico de las enfermedades del seno, se tocaron algunos tópicos relacionados, entre ellos el de la citología, habiendo causado impresión desfavorable mi opinión de que este procedimiento es aún poco confiable en nuestro medio.

Me propuse entonces revisar algunos artículos sobre este tema específico y quiero compartir el fruto de esas lecturas, lo cual no hace otra cosa diferente que complementar observaciones personales previas sobre la citología en general y sus limitaciones, $(1,2,3,4)$.

\section{DEFINICIONES}

Citología: Observación microscópica de las características de la célula.

Secreción, aspiración, impresión

Aspiración por aguja fina (F. N. A.).

\section{ANTECEDENTES}

Desde el punto de vista de la citología del seno pueden obtenerse varias muestras para estudio, Una por secreción: espontánea o provocada a través del pezón; otra por aspiración en procesos quísticos, o por punción de masas sólidas. También haciendo impresión de biopsias o especímenes quirúrgicos. Describiré cada uno de estos métodos, brevemente:

Patólogo Jefe de Laboratorio. Clínica Comuneros.

Bucaramanga

\subsection{Citología de Secreción por el pezón}

Aunque descrita por Papanicolau en 1958 (6) no fue muy popularizada por él, pues en su concepto, aparecido cinco años más tarde en su Atlas "las condiciones patológicas que causan secreción espontánea no son lo suficientemente numerosas para permitir un amplio uso de este método diagnóstico" (7). Esta opinión, aparentemente, fue compartida por Ruth Graham pues en su texto ni siquiera se menciona el estudio del seno por medio de la citología (8). Probablemente la creencia en la poca frecuencia de los procesos papilares dentro de la patología de la glándula mamaria, haya sido un factor importante, pues autoridades como Haagensen aún opinan "que el papiloma intracanalicular no es una enfermedad muy común" (9); sinembargo, es indudable que hoy día es mucho más frecuente la secreción del pezón en la mujer, pues las Displasias mamarias quísticas, la mayor parte de ellas con un componente papilar acentuado y los múltiples tratamientos médicos en la farmacopea moderna como los tranquilizantes y los anovulatorios entre otros, con frecuencia la determinan (10).

Según Ringrose "se obtiene secreción mamaria en el $27 \%$ de las mujeres premonopáusicas nuligrávidas y en el $40 \%$ de las premenopáusicas que han tenido hijos" sólo haciendo suave compresión de las mamas (11).

\subsection{Citología por aspiración}

No es un procedimiento nuevo en nuestro medio; sin exagerar se puede decir que llegó con el servicio de radioterapia a Bucaramanga pues fui testigo de la obtención de muchas muestras, por el ya desaparecido Oncólogo Dr. Francisco Espinel Salive en el antiguo Hospital San Juan de Dios, como 
lo ha reconocido al conmemorar los treinta años de la Anatomía Patológica en Bucaramanga; desafortunadamente esas experiencias nunca se recopilaron ni publicaron y por eso hoy día hay la equivocada impresión de que se está haciendo por primera vez entre nosotros (12).

\subsection{Citología por punción}

Las punciones de tumores en el ámbito mundial, fueron preconizadas por los hematólogos a comienzos del siglo XX y quizá alguién más las haya hecho antes (13).

El 1912 Ward aconsejaba la punción en ganglios linfáticos para el diagnóstico de linfomas; posteriormente Guthrie en 1921 comunicaba sus experiencias sobre las punciones en la enfermedad de Hodgkin y más tarde en 1926 Martín Ellis las rutinizaban en el Memorial Hospital de New York, en los tumores palpables.

Una modalidad de la punción general es la practicada con aguja fina, hoy tan en boga, no sólo para el diagnóstico de enfermedades del seno sino también tiroides, próstata (14) y otros órganos. Se considera que este método se usó por primera vez en 1904 por Griek y Gay quienes lo aplicaron para el diagnóstico de tripanosomiasis en los ganglios linfáticos (15).

\subsection{Citología por impresión}

Método más sofisticado desde el punto de vista de su interpretación que sólo compromete a patólogos. Puede tomarse la muestra para este estudio, del espécimen logrado por una biopsia incisional o excisional. También del material obtenido por punción; sin embargo, su uso rutinario como principal ayuda diagnóstica no es aconsejable. (16).

\section{TECNICA}

No es mi intención describir los diferentes pasos, ni el equipo a emplear en la toma del material para estudio. Cuando hay secreción puede ser lograda por la misma paciente o en el consultorio, por el médico, la enfermera o el citólogo; lo importante es la rápida fijación.

En el caso de la punción por aspiración, la muestra debe ser tomada por un profesional con experiencia en ese campo y entrenamiento adecuado para evitar demasiados aspirados no diagnósticos o insuficientes. Algunas escuelas europeas teniendo en cuenta que en nuestro medio la mayoría de las veces no funciona "la estrecha y fuerte relación profesional entre el patólogo y el clínico" abogan por el "Citopatólogo intervencionista" (17). Debe sí borrarse la falsa impresión que la Citología por aspiración de la mama por lo aparentemente fácil de la técnica para tomar la muestra y "la sencillez del instrumental con que se efectúa, puede ser realizada en todas partes y por cualquiera" (13). Es, si, un hecho indudable que practicada con todos los requisitos, su economía en tiempo y dinero, el mínimo grado de malestar y de complicaciones que causan al paciente y por no requerir preparación alguna en éste, habrán de hacerla más utilizable cada día.

Se sugiere que los resultados sean informados como positivas, negativas, dudosa como se hace con otras muestras diferentes para evitar confusiones en la interpretación (18).

\section{APLICACIONES}

Es indudable que de las citologías la por aspiración es una de las técnicas que inevitablemente será más utilizada en el campo de la Anatomía Patológica y que siempre tendrá opositores entre "los patólogos clásicos que a toda costa exigirán la remisión de cualquier tipo de tejido" (15).

Aunque podría hacerse una larga lista sobre sus indicaciones, quizá sea más práctico decir que puede practicarse en todo proceso palpable mayor de 1 $\mathrm{cm}$, profundo "pero sobre todo en aquellos en los cuales la extracción de una muestra presenta dificultad o supone gran esfuerzo”. (13).

Es importante sí, usar la técnica apropiada.

\section{CONFIABILIDAD}

Este procedimiento de laboratorio en manos adecuadas da una certeza del $96 \%$. Sin embargo; una revisión de trabajos citada por Schondorf da cifras de falsos negativos que oscilan entre el 3 y el $24 \%$ y falsos positivos entre el 0.1 y el $9 \%$. No obstante para que sea exitoso, es preciso, así como en la aspiración biopsia por aguja fina (F. N. A.) "una exquisita capacidad técnica del operario y del analizador" (15). Sin estos dos requisitos resultará decepcionante y traerá como consecuencias, diagnósticos erróneos, con graves inconvenientes. Hay quienes opinan que en un entrenamiento o experiencia en la interpretación de un extendido implica una lectura mínima de 100 casos ya diagnosticados en un centro, antes de aventurarse a emitir la propia opinión en un nuevo paciente. En el I. S. S. en Bu- 
caramanga hasta este momento se tienen estudiados 35 casos en cinco años que corresponden a secreciones o punciones. El número tan reducido de nuestros pacientes no nos permite aportar comentario alguno. Sólo hemos observado la importancia del procesamiento rápido y adecuado de las muestras con el fin de obtener preparaciones de buena calidad que no compliquen su interpretación. Esta no es muy sencilla, pero tampoco demasiado difícil.

\section{CONCLUSIONES}

La Citología como método de diagnóstico en las enfermedades del seno constituye una técnica sencilla, económica en tiempo y dinero, que causa mínimo grado de molestia en la toma y que puede repetirse. No tiene contraindicación alguna, fuera de las de toda punción y la única complicación posible es la inflamación.

Requiere de un buen técnico, un experimentado analista y una estrecha comunicación entre ellos; sólo en estas condiciones dará un acierto hasta del $97 \%$, pues de lo contrario pueden lograrse aspirados no diagnósticos, insuficientes o inadecuados. Un informe citológico negativo no descarta totalmente la posibilidad de una lesión maligna; un diagnóstico citológico de malignidad, no debe ser la única base para una terapia agresiva, médica o quirúrgica.

\section{BIBLIOGRAFIA}

1. GAMbOA G. AlVARO, CORTES C. Carlos et al, Tendencia al Cáncer Genital en Bucaramanga. Tribuna Médica No. 60 a 15-18 mayo de 1975.

2. CORTES C.C.; ACEVEDO, S.A. Citología Exfoliativa. LaboLaboratorio Clínico Patológico. Boletín Informativo No. 2 Agosto 1981.

3. CORTES C. CARLOS, Citología Exfoliativa. Laboratorio Clínico y Anatomía Patológica. Boletín Informativo No. 6 Marzo 1983.

4. CORTES C. CARLOS, CORREA DE REYES, ELSA. Citología y Cáncer Genital. Revista Medicina. No. 20 Pg. 22-23 1989.

5. CARTER DARRYH. Interpretation of breast biopsies, Pg. 12 -17 Raven Press. New York, 1984.

6. PAPANICOLAU GEORGE N. et al. Exfoliative Cytology of the Human mamary gland and its value in the diagnosis of cancer and other diseases of the breast, Cancer 11-377-409.

7. PAPANICOLAU GEORGE N. Atlas of Exfoliative Cytology, Pag. 54 Cambridge. Massachusetts, Harvard, University 1963.

8. GRAHAM RUTH. The Cytology of Cancer-Second Edittion, W.B. Sanders Company, Philadelphia and London 1963.

9. HAAGENSEN C.D. Enfermedades de la mama, Pag. 159 Tercera Edición Panamericana 1987.
10. DE AZUA, BLANCo JAVIER. Citología por Punción. Aspiración con aguja fina, Pag.134. Salvat Editores 1987.

11. RINGROSE (Citado por) TAKAHASHI MASAYOSHI-, Atlas Color, Citología del Cáncer, pág. 243. Editorial Médica Panamericana, Segunda Edición. Abril 1982.

12. CORTES C. CARLOS. Treinta años de Anatomía Patológica en Bucaramanga, Sesión Academia de Medicina. Diciembre 9. 1988.

13. SCHONDORF HUBERT. Citología por aspiración de la mama, Salvat 1979.

14. MENDOZA F. LUPI SERGIO. Punción Aspiración con aguja fina de la Próstata. Presentación de casos. Bucaramanga, Marzo 10 de 1989. Universidad del Valle.

15. MIRALLES T.G.;BUESA J.M. Punción aspiración con aguja fina. Salvat 1988.

16. HUNGHES AND DODDS. Handbook of Diagnostic Cytology E\&S Livingstone Ltda. Edinburgh and London 1968 (pag. 153).

17. GROHS HINZ K M DF A C. The interventional Cytopathologyst. American Journal of Clinical Pathology. Vol. 90 - 3 Pag 351-54 September 1988.

18. GAYA G. JAMES et col. Transthoracic Fine-needle Aspiration Cytology. Am. J. Clin Pathol 82:100-103. 1984.

\section{* FOCUS: LA PLANIFICACION FAMILIAR SALVA VIDAS}

Nota a los lectores:

La Organización Mundial de la Salud (OMS) ha estado colaborando con la Federación Internacional de Ginecología y Obstetricia (FIGO), a través de uno de sus grupos de trabajo denominado TASK FORCE, en Salud Materno Infantil y Planificación Familiar, en la atención primaria de la salud desde 1983.

Una de las actividades de este grupo conjunto de trabajo ha sido el desarrollo de una serie de artículos denominados FOCUS, el primero de los cuales aparece en la página siguiente de esta revista.

Esta serie tratará en forma concisa de reportar un problema particular, las formas de abordarlo conduciendo hacia su resolución e indicando los tipos de acciones que pueden ser utilizados por los profesionales de la salud para obtener los resultados beneficiosos. 